\title{
Relaciones bidireccionales longitudinales entre victimización, estilos inferenciales de desesperanza y síntomas de depresión en adolescentes: Un modelo transaccional
}

\author{
Eneko Ruiz-Alonso, Izaskun Orue y Esther Calvete
}

Universidad De Deusto, Bilbao, España

\begin{abstract}
Longitudinal bidirectional relationships between victimization, inferential styles of hopelessness, and symptoms of depression in adolescents: A transactional model
\end{abstract}

\begin{abstract}
Adolescent depression is a very common problem that has serious consequences. The present study examines the bidirectional relationships between depression, inferential styles of hopelessness and peer victimization. The sample consisted of 1325 students ( 597 females, 726 males, and 2 who did not indicate their gender, aged 12 to 17 years, Mage $=14.16 ; S D=1.26$ ). Participants completed measures of depression, peer victimization and inferential styles of hopelessness on three occasions each separated by 6 months. Results show that depressive symptoms predict a worsening of inferential styles, which in turn produce an increase in depressive symptoms. Likewise, peer victimization predicts an increase in depressive symptoms. The findings support a transactional model with reciprocal relationships between depressive symptoms and inferential styles of hopelessness.
\end{abstract}

Keywords: Peer victimization; inferential styles; depression; adolescents.

Resumen: La depresión en la adolescencia es un problema común que genera graves consecuencias. El presente estudio examina las relaciones bidireccionales existentes entre la depresión, los estilos inferenciales de desesperanza y la victimización entre iguales. La muestra consistió en 1325 estudiantes ( 597 chicas, 726 chicos y 2 que no indicaron su género, de entre 12 y 17 años, edad media $=14.16 ; D T=1.26)$. Los participantes completaron medidas de depresión, victimización entre iguales y de estilos inferenciales de desesperanza en tres momentos con un distanciamiento de 6 meses entre los mismos. Los resultados muestran que la sintomatología depresiva predice un empeoramiento de los estilos inferenciales, los cuales a su vez producen un aumento de la sintomatología depresiva. Así mismo, la victimización entre iguales predice un aumento de la sintomatología depresiva. Los hallazgos respaldan un modelo transaccional en el que resaltan las relaciones recíprocas entre síntomas depresivos y estilos inferenciales de desesperanza.

Palabras clave: Victimización entre iguales; estilos inferenciales; depresión; adolescentes.

\section{Introducción}

La adolescencia es un periodo de grandes cambios físicos, sociales y psicológicos, por lo que es considerada

Recibido: 26 de octubre 2020; aceptado 09 de junio de 2021

Correspondencia: Eneko Ruiz-Alonso, Facultad de Psicología, Universidad de Deusto, Avenida de las Universidades, 24, 48007 Bilbao, España. Correo-e: ruiz.eneko@deusto.es

Agradecimientos: Este proyecto ha sido financiado por el Gobierno Vasco (IT982-16) como un periodo crítico para el desarrollo de diferentes enfermedades mentales y, en particular, de los trastornos depresivos (Hankin et al., 1998). Entre las consecuencias de estos trastornos depresivos en la adolescencia destacan los problemas interpersonales, académicos y riesgo de autolesiones entre otros (Marshall et al., 2013; Moore et al., 2017). Por ello, numerosas investigaciones han tratado de dar una explicación al desarrollo de la depresión durante la adolescencia. Uno de los modelos más estudiados y con mayor validez obtenida ha sido el modelo de diátesis-es- 
trés, que explica que los estresores junto a los estilos cognitivos son los precursores de los síntomas depresivos (Calvete et al., 2013; Mac Giollabhui et al., 2018).

Uno de los estresores más relevantes en la adolescencia es la victimización entre iguales. La victimización es un grave problema en los centros escolares. Los estudios meta-analíticos muestran que entre el $8 \%$ y el $43 \%$ de los estudiantes sufren o han sufrido victimización crónica, siendo su mayor pico en los años de secundaria, con niveles similares para chicos y chicas (Hymel y Swearer, 2015; Pouwels et al., 2016). Numerosos estudios alertan de las graves consecuencias que este tipo de victimización puede acarrear. Por ejemplo, un estudio mostró que los niños y jóvenes que sufren de victimización desarrollan entre otras, dificultades de ajuste, ansiedad social, depresión, estrés y cambios en los esquemas cognitivos (Calvete et al., 2018).

Uno de los estilos cognitivos que más atención ha recibido en el desarrollo de depresión en adolescentes es el de la desesperanza (Abramson et al., 1989). Acorde con la teoría de la desesperanza de Abramson et al. (1989), los síntomas depresivos están determinados en buena medida por los estilos inferenciales negativos, dentro de los que se incluyen (a) tendencia a atribuir eventos negativos de forma interna, global y estable, (b) tendencia a percibir los eventos negativos como si fuesen a tener unas consecuencias mucho mayores de las que verdaderamente van a tener y (c) tendencia a desarrollar inferencias negativas sobre uno mismo durante los eventos negativos. La teoría de la desesperanza, encuadrada en los modelos diátesis-estrés, explica que los estilos cognitivos influyen de manera directa en el desarrollo de depresión y en la forma en la que esta puede transformarse en crónica y severa (Rose y Abramson, 1992). Los estilos inferenciales incrementan en estabilidad a lo largo de los años y tienden a volverse relativamente estables en la adolescencia (Cole et al., 2008; Hankin, 2008).

Se han realizado numerosos estudios para examinar en qué medida los estilos inferenciales de desesperanza solos o en interacción con circunstancias estresantes como la victimización predicen la depresión en adolescentes (Gibb y Abela, 2008; Mateos-Pérez y Calvete, 2019). Además, tiene lugar cierta especificidad en el sentido de que los estilos inferenciales referidos a acontecimientos sociales son más relevantes para experiencias sociales negativas como el bullying. Así, por ejemplo, un adolescente que tiene un estilo inferencial de desesperanza considerará que no puede hacer nada por evitar las conductas de victimización, generando un estado de indefensión, lo que facilitará que estas conductas se sigan produciendo (Mateos-Perez y Calvete, 2019).

Por otro lado, las relaciones entre los estilos inferenciales de desesperanza, la victimización entre iguales y la sintomatología depresiva pueden ser complejas y bidireccionales, tal y como explica el modelo transaccional cognitivo de la vulnerabilidad al estrés de Hankin y Abramson (2001). Este modelo va más allá del modelo diátesis-estrés tradicional y propone que los estilos cognitivos, las circunstancias estresantes y la depresión se influyen mutuamente. En otras palabras, propone que las relaciones entre las cogniciones, los síntomas depresivos y los estresores son dinámicas y bidireccionales. Así, este modelo enfatiza la importancia de las relaciones bidireccionales, sugiriendo que en ocasiones las personas entran en una espiral en la que todos estos factores se influyen los unos a los otros.

A partir de esta teoría han aparecido otros modelos como el modelo de desarrollo de cascada (Masten et al., 2005). Este modelo sugiere que: (1) los estresores y los estilos inferenciales desadaptativos incrementan la probabilidad de sufrir síntomas depresivos. (2) Una vez los síntomas depresivos aumentan, junto con los estresores, producen un empeoramiento en los estilos inferenciales. (3) Tras el empeoramiento de los estilos inferenciales, junto con los síntomas depresivos, se produce un aumento en el número de estresores. Así, todos estos mecanismos contribuyen a producir un efecto cascada, influyéndose los unos a los otros. Es por este motivo por lo que generalmente estas problemáticas aparecen de forma conjunta en los adolescentes y no de forma aislada (Calvete et al., 2013; Hankin y Abramson, 2001; Masten et al., 2005). Sin embargo, han sido pocos los estudios que han tratado de comprobar estos modelos teóricos y por tanto estas relaciones bidireccionales entre la victimización entre iguales, los estilos inferenciales de desesperanza y síntomas depresivos, que son el foco del actual estudio. La Figura 1 muestra el modelo conceptual que guía este estudio. Los mecanismos implicados incluyen los fenómenos de generación de estrés y de empeoramiento cognitivo, además de las tradicionales asociaciones predictivas entre estresores y estilos cognitivos, por un lado, y síntomas de depresión, por otro.

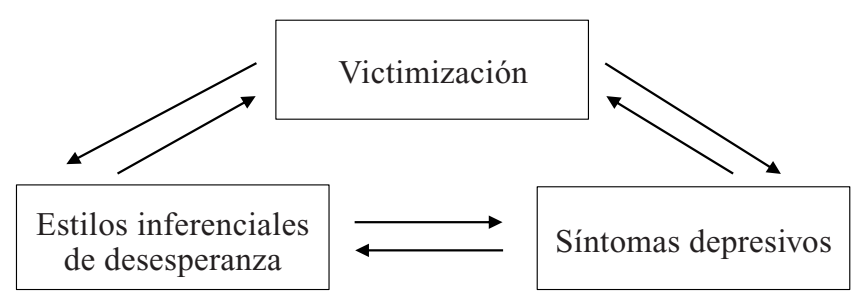

Figura 1. Modelo conceptual de relaciones bidireccionales entre victimización, estilos inferenciales y síntomas depresivos.

La hipótesis de la generación del estrés hace referencia al hecho de que las personas deprimidas contribuyen a la generación de situaciones estresantes adicionales en 
sus vidas. Como consecuencia de niveles previos de depresión, el número de estresores aumenta, y estos a su vez aumentan los síntomas depresivos (Hammen, 2005). Diversos estudios han confirmado la existencia del modelo de generación de estrés en muestras de niños y adolescentes (Saint-Georges y Vaillancourt, 2020; Zhang et al., 2020). Como se ha mencionado anteriormente, uno de los mayores estresores en la adolescencia es la victimización entre iguales. Así, siguiendo el modelo de generación del estrés de Hammen, se podría considerar que el sufrir depresión aumentaría la probabilidad de sufrir victimización, teniendo siempre en cuenta que esta relación podría ser a su vez bidireccional. Por ejemplo, un adolescente que es victimizado podría como consecuencia experimentar un estado de ánimo depresivo, y este a su vez podría afectar negativamente a sus relaciones con iguales, contribuyendo a nuevas victimizaciones. Este fenómeno ha sido confirmado en estudios longitudinales como el de Zhang et al. (2020), en el que los síntomas depresivos estaban relacionados de forma directa con la victimización por cyberbullying.

Más aún, el modelo de generación de estrés incluye también los estilos cognitivos como antecedentes de estrés, explicando que estos estilos cognitivos podrían aumentar el riesgo de nuevas victimizaciones. Dentro de los estilos cognitivos se ha estudiado el papel que juegan los esquemas maladaptativos tempranos y la rumiación en la generación de estrés (Alba et al., 2018). Por ejemplo, Janovsky et al. (2020), en su estudio meta-analítico, manifestaron la existencia de una relación directa entre los esquemas maladaptativos tempranos y los problemas interpersonales entre los que se encuentra la victimización. Mientras que, por otro lado, estudios longitudinales como el de Calvete et al. (2013) destacaron a los estilos inferenciales como grandes precursores de situaciones estresantes.

Otro mecanismo implicado en el modelo transaccional es el del empeoramiento cognitivo (Calvete et al., 2013). Este mecanismo explica que los síntomas depresivos predicen un empeoramiento en las vulnerabilidades cognitivas incluyendo los esquemas cognitivos o la rumiación entre otras, lo que ha sido remarcado por un gran número de estudios (Davoodi et al., 2018; Krause et al., 2018). Incluso enmarcados dentro de este modelo de empeoramiento, se encuentran estudios como el de Calvete (2011), que encontraron que la sintomatología depresiva producía un empeoramiento directo en los estilos inferenciales. Posteriormente se determinó también que las circunstancias estresantes podían generar un empeoramiento en los estilos cognitivos (Calvete et al., 2015). Por ejemplo, el estudio longitudinal de Cole et al. (2016) determinó en una muestra de 827 chicos y chicas que el sufrir victimización estaba relacionado directamente con un empeoramiento en las cogniciones negativas autorreferenciales, demostrando así las graves consecuencias que tiene el sufrir conductas de victimización sobre las cogniciones de las personas.

El presente estudio pretende extender las investigaciones existentes al examinar las relaciones bidireccionales entre la victimización entre iguales, los estilos inferenciales de desesperanza y los síntomas depresivos. En contraste con la mayoría de los estudios anteriores que han examinado sólo alguna de estas relaciones, el estudio actual evalúa tres posibles relaciones entre estas variables de manera simultánea: (1) estilos inferenciales de desesperanza y victimización entre iguales como predictores de los síntomas depresivos (modelo de la vulnerabilidad), (2) síntomas depresivos y estilos inferenciales de desesperanza como predictores de la victimización entre iguales (modelo de generación del estrés), y (3) síntomas depresivos y victimización entre iguales como predictores de los estilos inferenciales de desesperanza (modelo de empeoramiento cognitivo). Se esperó encontrar la existencia de relaciones bidireccionales entre los estilos inferenciales de desesperanza, la victimización entre iguales y la sintomatología depresiva, produciéndose un efecto cascada similar al propuesto por Masten et al. (2005).

\section{Método}

\section{Participantes}

La muestra total del estudio fue de 1467 adolescentes que fueron invitados a participar de manera voluntaria en el estudio. Los adolescentes pertenecían a 51 aulas de 8 centros educativos de Bizkaia (España). Las diferentes escuelas fueron seleccionadas al azar de una lista de escuelas disponibles en Bizkaia. De los 1467 adolescentes invitados, 1316 participaron en el T1, 1306 en el T2 y 1179 en el T3. De todos los participantes, 1325 (597 chicas, 726 chicos y 2 sujetos que no han indicado su género) completaron medidas en al menos dos de los tres tiempos del estudio (tasa de participación: 90.32\%). La tasa de abandono fue debida principalmente a la ausencia de los adolescentes en la escuela debido a enfermedades y a errores en los códigos empleados para emparejar las medidas en los tres tiempos. Los participantes tenían entre 12 y 17 años de edad al comienzo del estudio $(M=14.16 ; D T=1.26)$. El nivel socio-económico fue determinado a través del criterio recomendado por la Sociedad Española de Epidemiología (2000) para la educación parental y los ingresos. Los niveles socio-económicos siguieron la siguiente distribución: $21.3 \%$ bajo, $14.3 \%$ medio-bajo, $27 \%$ medio, $18.1 \%$ medio-alto, $19.3 \%$ alto. 


\section{Procedimiento}

El Comité de Ética de la Universidad de Deusto aprobó este estudio. Tras lo cual, se contactó con las escuelas por teléfono y se explicó el estudio para obtener su permiso. La participación fue voluntaria y no se incluyeron nombres en los cuestionarios. Los adolescentes fueron informados de que sus respuestas eran confidenciales y sólo serían empleadas por el equipo de investigación. Los progenitores fueron informados y se les dio la opción de negarse a permitir la participación de sus hijos. Los adolescentes completaron los cuestionarios en sus aulas en tres puntos de tiempo diferentes, estando estos separados por 6 meses de diferencia entre ellos. Todos los cuestionarios se completaron en cada uno de los tiempos. Para emparejar los cuestionarios de T1, T2 y T3, se empleó un código solo conocido por el participante. Los psicólogos del equipo de investigación fueron los encargados de la recopilación de los datos en las aulas.

\section{Instrumentos}

Cuestionario de Estilos Cognitivos de Adolescentes (ACSQ; Hankin y Abramson, 2002; versión en español de Calvete et al., 2007). Este cuestionario mide la vulnerabilidad cognitiva incluyendo inferencias negativas hacia las causas de los eventos negativos, inferencias negativas sobre sus consecuencias y las implicaciones para uno mismo. El cuestionario presenta diferentes escenarios hipotéticos. En este estudio se utilizaron tres escenarios de naturaleza interpersonal (p.ej., «No has sido invitado a una fiesta de cumpleaños de un amigo»). Se pide a los adolescentes que imaginen cada uno de estos hipotéticos escenarios y evalúen en qué medida las causas de estos escenarios son internas, estables y globales, sus consecuencias y en qué medida este acontecimiento implica que algo falla en ellos. La escala de respuesta varía de entre 1 y 7 , y los valores más altos indican unos estilos cognitivos negativos y los valores bajos estilos cognitivos positivos. Varios estudios longitudinales han demostrado la validez y la fiabilidad del ACSQ como medida de los estilos inferenciales de la versión original (Cole et al., 2008; Hankin, 2008) y en la versión española (Calvete et al., 2008). En el presente estudio, para la puntuación total del estilo inferencial, el coeficiente alpha en el T1 ha sido de .89, en el T2 de .91 y en el T3 de .92.

Escala del Centro para el Estudio Epidemiológico de la Depresión (CES-D; Radloff, 1977; versión en español de Calvete y Cardeñoso, 1999). Esta escala mide cuatro factores de la depresión (disminución psicomotora, afecto positivo, dificultades interpersonales y afecto depresivo) y permite obtener una puntuación global de síntomas de depresión. El cuestionario incluye 20 ítems, con opciones de respuesta que oscilan entre 0 (prácticamente nunca) y 3 (casi todo el tiempo). Una puntuación mayor de 16 indicaría un estado de ánimo depresivo leve, mayor de 23 indicaría un estado de ánimo depresivo moderado, finalmente una puntuación igual o superior a 28 indicaría un estado de ánimo depresivo grave consistente con un trastorno depresivo mayor. La versión española del CES-D presenta unas propiedades psicométricas excelentes (Calvete y Cardeñoso, 1999). En este estudio, el coeficiente alpha en el T1 ha sido .79, en el T2 .81 y .79 en el T3.

Escala de Victimización del Cuestionario de las Relaciones entre Iguales para Niños (PRQ; Rigby y Slee, 1993; versión en español de Calvete, 2014). La Escala de Victimización incluye 7 ítems, uno de ellos describe la victimización física (p.ej., «Ellos me pegan o me empujan») y el resto de los ítems evalúan la victimización emocional (p.ej., «Ellos abusan de mí en la escuela», «me excluyen de los grupos aposta»). Los participantes tienen que indicar la frecuencia con la que ocurren este tipo de acciones en los últimos 6 meses en una escala de 4 puntos, siendo 1 (No me ocurre) y 4 (Me ocurre muy a menudo). La versión española se desarrolló mediante un procedimiento de traducción y retrotraducción, y su estructura fue confirmada (Calvete, 2014). Los diferentes estudios que han empleado esta herramienta han demostrado que esta es una medida adecuada de la experiencia de la victimización entre iguales con buena validez y fiabilidad (Bond et al., 2007). En el actual estudio el coeficiente alpha en el T1 ha sido .83, en el T2 .85 y en el T3 .83.

\section{Análisis estadísticos}

Se utilizó path analysis con MPLUS 8.2 con el método de estimación robusto de máxima verosimilitud. Mplus utiliza FIML (full information maximun likelihood) para el manejo de los valores perdidos. Siguiendo las recomendaciones de varios autores (Hu y Bentler, 1999; Little, 2013), la bondad del ajuste se evaluó utilizando el índice de ajuste comparativo (CFI), el índice de ajuste no normativo (NNFI), el error cuadrático medio de aproximación (RMSEA), y la raíz cuadrada media residual estandarizada (SRMR). En general, los valores CFI y NNFI de 0.95 o más altos y los valores RMSEA y SRMR inferiores a 0.06 y 0.08 , respectivamente, indican un buen ajuste para los modelos longitudinales (Little, 2013). 


\section{Resultados}

Análisis descriptivos y correlaciones entre las variables

En cuanto a los síntomas depresivos en el T1 un $28.3 \%$ de los participantes experimentaron síntomas de depresión leves, un $8.7 \%$ experimentaron síntomas moderados de depresión y un $12.3 \%$ experimentaron síntomas graves de depresión. En el T2 el porcentaje fue de $21.8 \%, 7.5 \%$ y $10.7 \%$, respectivamente, y en el T3 fue de $21.1 \%, 7.4 \%$ y $10.3 \%$. Por otro lado, en lo que respecta a la victimización entre iguales, alrededor de la mitad de los adolescentes habían sufrido al menos un acto de victimización en los últimos 6 meses. En concreto, el porcentaje de participantes que habían sido víctimas de algún tipo de comportamiento de victimización fue de $57.6 \%$ en $\mathrm{T} 1,52.8 \%$ en $\mathrm{T} 2$, y $48.1 \%$ en T3.

La Tabla 1 refleja los coeficientes de correlación entre todas las variables del estudio, así como las medias y las desviaciones estándar para cada uno de los tiempos T1, T2 y T3. Como se puede ver, todos los coeficientes fueron estadísticamente significativos, $p<.001$, y varios de ellos fueron particularmente altos $(r \geq .6)$.

Tabla 1. Coeficientes de correlación entre las variables del estudio

\begin{tabular}{|c|c|c|c|c|c|c|c|c|c|c|}
\hline & 1 & 2 & 3 & 4 & 5 & 6 & 7 & 8 & $M$ & $D T$ \\
\hline 1. T1 Depresión & & & & & & & & & 17.64 & 8.95 \\
\hline 2. T1 Victimización & .33 & & & & & & & & 0.30 & 0.44 \\
\hline 3. T1 Desesperanza & .34 & .20 & & & & & & & 2.83 & 1.02 \\
\hline 4. T2 Depresión & .59 & .27 & .33 & & & & & & 16.17 & 9.46 \\
\hline 5. T2 Victimización & .27 & .65 & .15 & .37 & & & & & 0.28 & 0.45 \\
\hline 6. T2 Desesperanza & .30 & .18 & .59 & .41 & .23 & & & & 2.83 & 1.05 \\
\hline 7. T3 Depresión & .53 & .25 & .28 & .66 & .25 & .34 & & & 15.74 & 9.37 \\
\hline 8. T3 Victimización & .23 & .57 & .13 & .27 & .61 & .18 & .32 & & 0.25 & 0.40 \\
\hline 9. T3 Desesperanza & .27 & .13 & .51 & .31 & .12 & .62 & .40 & .16 & 2.85 & 1.05 \\
\hline
\end{tabular}

Nota. Todos los coeficientes son estadísticamente significativos $\mathrm{p}<.001$.

\section{Modelo mediacional hipotético}

En primer lugar, se estimó el modelo predictivo, que incluyó asociaciones transversales entre todas las variables del T1, y entre los residuos de las variables del T2 y entre las variables del T3, asociaciones auto-regresivas que representan la estabilidad de las medidas, y asociaciones predictivas cruzadas entre todas las variables. Los resultados mostraron que la victimización entre iguales, el estilo de inferencias y los síntomas de depresión en el T1 estaban asociados significativamente de forma positiva entre sí. Además, el sexo masculino se asoció negativamente con los síntomas de depresión y marginalmente de forma positiva con la victimización. La edad se asoció negativamente con la victimización. Además, todas las asociaciones auto-regresivas fueron estadísticamente significativas.

En cuanto a las relaciones cruzadas longitudinales entre variables, los resultados mostraron que el estilo de inferencias de desesperanza predecía los síntomas de depresión del T1 al T2 y del T2 al T3 y que, a su vez, los síntomas de depresión predecían el estilo de desesperan- za del T1 al T2 y del T2 al T3. Además, la victimización en el T1 predijo los síntomas de depresión en el T2 y los síntomas de depresión en el $\mathrm{T} 1$ predijeron la victimización el T2. Finalmente, el sexo masculino predijo un menor aumento de depresión del T1 al T2 y un mayor aumento de victimización del T1 al T2.

Los índices de modificación indicaron que el modelo mejoraba añadiendo path directos de victimización en el $\mathrm{T} 1$ a victimización en el T3 por lo que este path fue incluido en el modelo. Los indicadores de ajuste para el modelo fueron moderados: $\chi^{2}(17, \mathrm{~N}=1325)=172, p<$ $.001, \mathrm{RMSEA}=.083(90 \%$ CI $[.072, .095]), \mathrm{TLI}=.927$, y CFI $=.964$, SRMR $=.029$. Se estimó un modelo más parsimonioso en el que se eliminaron los parámetros que no eran estadísticamente significativos. Los índices de ajuste fueron excelentes: $\chi^{2}(22, \mathrm{~N}=1325)=124, p<$ $.001, \mathrm{RMSEA}=.059$ (90\% CI $[.049, .069]), \mathrm{TLI}=.856$, y CFI $=.956$, SRMR $=.038$. La Figura 2 muestra el modelo estimado. Los resultados sugieren que el estilo de inferencias de desesperanza en el T2 media entre depresión en el T1 y depresión en el T3 y que, a su vez, la depresión en el T2 media entre el estilo de inferencias de 
desesperanza en el T1 y en el T3. Se realizó un procedimiento de bootstrapping con 5000 muestras y los resultados apoyaron estos efectos mediacionales, dado que los intervalos de confianza no incluyeron el cero para depresión en el T1 a depresión en el T3 (0.010, IC5\%/95\% $=0.05$
-0.018 ) y para estilo de inferencias en el T1 a estilo de inferencias en el T3 $(0.011,(\mathrm{IC} 5 \% / 95 \%=0.004-0.020)$. El modelo final explicó un porcentaje de la varianza de $45.5 \%$ de depresión, $43.2 \%$ de victimización y $38.7 \%$ de estilo de desesperanza en el T3.

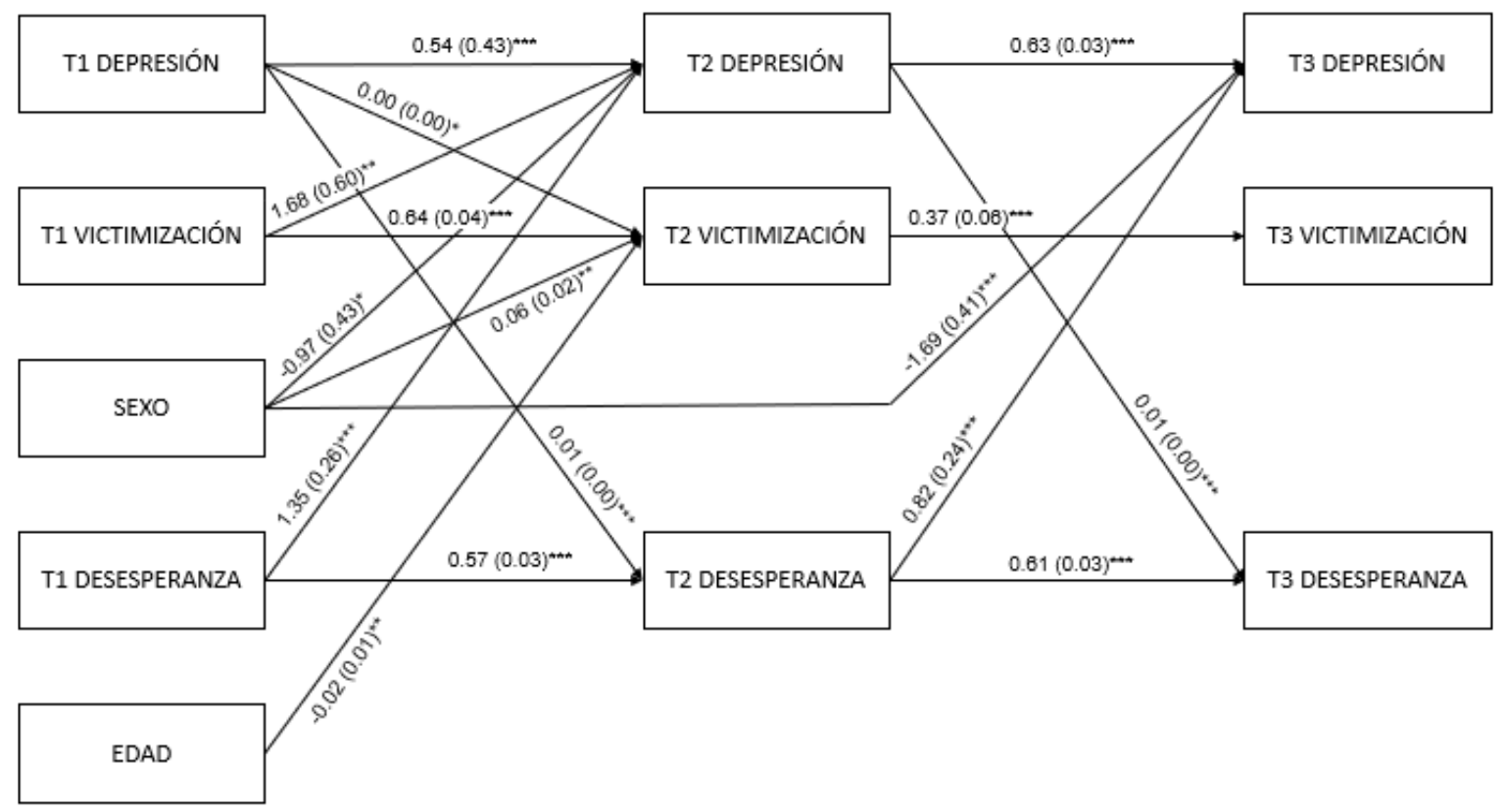

Figura 2. Paths longitudinales entre depresión, victimización, sexo, desesperanza y edad. Se muestran los coeficientes no estandarizados con los errores típicos entre paréntesis. $T 1=$ Tiempo $1, T 2=$ Tiempo $2, T 3=$ Tiempo $3,{ }^{*} p<.05 ; * * p<.01 ; * * p<.001$.

\section{Discusión}

La depresión en la adolescencia es un problema muy habitual, generando graves consecuencias en la salud física y mental de los jóvenes. Identificar qué factores están implicados en la aparición de la depresión es fundamental para poder prevenir la aparición de la sintomatología depresiva y elaborar programas de intervención eficaces. En cuanto a la prevalencia de la depresión y la victimización, se ha mostrado la elevada prevalencia de ambos, coincidiendo así con otros estudios como el de Alba et al. (2018) o el de Calvete et al. (2015) que muestran el nivel elevado de conductas de victimización a las que se ven expuestos los adolescentes y la alta sintomatología depresiva de los mismos.

Estudios previos al actual muestran cómo los estilos inferenciales y los estresores favorecen la aparición y el desarrollo de la sintomatología depresiva (Calvete et al., 2015; Mac Giollabhui et al., 2018). Así, el presente estudio se basa en los modelos transaccionales de la psicopatología y examina las relaciones bidireccionales entre la sintomatología depresiva, la victimización entre iguales y los estilos inferenciales de desesperanza.
En lo que se refiere a la predicción de síntomas depresivos, los estilos inferenciales predijeron un aumento de los síntomas depresivos. A pesar de la escasa existencia de estudios longitudinales que hayan analizado el papel de los estilos inferenciales, los resultados del actual estudio son acordes con diversos estudios que remarcan el papel de las cogniciones en la aparición de la depresión (Alba y Calvete, 2019; Gómez-Odriozola y Calvete, 2020). Por otro lado, el estudio actual muestra como el haber sufrido victimización produce un aumento de los síntomas depresivos, lo que es acorde con el modelo diátesis-estrés, explicando que la victimización entre iguales es uno de los estresores que con mayor frecuencia experimentan los adolescentes, y que este aumenta considerablemente el riesgo de desarrollar síntomas depresivos, lo que ha sido refutado por diversos estudios (Bowes et al., 2016; Silva et al., 2020). Sin embargo, en este estudio, la predicción solo tuvo lugar entre el T1 y el T2 dado que la victimización en el T2 no predijo aumento de depresión en el T3. En cuanto al género, al igual que en meta-análisis como el de Salk et al. (2017), el actual estudio recoge que las mujeres tienden a aumentar sintomatología depresiva más que los hom- 
bres, estando esta relación existente del T1 al T2 y del T2 al T3.

Como Lewinsohn y sus colegas (1981) propusieron en el modelo de empeoramiento cognitivo, el actual estudio muestra cómo la sintomatología depresiva predice un empeoramiento de los estilos inferenciales, haciendo que los adolescentes adquieran una visión pesimista de sí mismos, consideren que las consecuencias van a ser mucho peores de lo que en realidad son y tengan inferencias negativas hacia las causas de estos eventos. Estos resultados van más allá, puesto que como se ha mostrado anteriormente, las relaciones entre los estilos inferenciales de desesperanza y la depresión son bidireccionales, lo que puede generar un efecto cadena o cascada como propusieron Hankin y Abramson (2001). En este efecto cascada, el aumento de los niveles de depresión produce estilos inferenciales desadaptativos, que a su vez producen un aumento de esa sintomatología depresiva, entrando en un bucle del que es difícil salir, de ahí la importancia que tienen los estudios como el actual, haciendo ver la necesidad de prevenir e intervenir para poder paliar este tipo de problemáticas.

Por otro lado, estudios como el de Calvete et al. (2015) o el de Cole et al. (2016), muestran cómo la victimización produce un empeoramiento de los estilos inferenciales, llevándolos a niveles disfuncionales. Sin embargo, los resultados del actual estudio no muestran la existencia de la relación predictiva entre victimización entre iguales y los estilos inferenciales de desesperanza.

El actual estudio, corroborando al fenómeno que Hammen (1991) exponía en su teoría de generación del estrés, muestra que la sintomatología depresiva en el T1 se relaciona de forma directa con la victimización entre iguales en el T2. Sin embargo, esta relación no estuvo presente del T2 al T3. Este estudio a diferencia de otros estudios longitudinales ha empleado como evento estresor la victimización entre iguales. Así, estos resultados concuerdan con otros estudios longitudinales que han empleado la victimización como medida general, mostrando que la sintomatología depresiva predice un aumento de los niveles de victimización (Sweeting et al., 2006; Zhang et al., 2020). Del mismo modo, el estudio actual muestra como la edad y el género se relacionan de forma directa con la victimización, explicando que los chicos son más propensos a sufrir conductas de victimización que las chicas, a pesar de que estudios previos al actual muestran que las chicas tienden a ser más propensas a la victimización (Chocarro y Garaigordobil, 2019; Delgado y Escortell, 2018).

Por último, hay que destacar que todas las variables empleadas en el estudio han mostrado tener propiedades de estabilidad en el tiempo. De ahí la importancia de la prevención temprana, ya que una vez se inicia este proceso complejo y dinámico entre la victimización, los estilos inferenciales desadaptativos y la depresión, los efectos tienden a acumularse y aumentar con el tiempo (Alba y Calvete, 2019; Calvete et al., 2015; Hankin, 2010).

El patrón de hallazgos debe considerarse a la luz de las fortalezas y debilidades del estudio actual. Así, entre los puntos fuertes de este estudio se incluye el empleo de una gran cantidad de participantes de diversos centros educativos que representan un amplio rango socioeconómico, o la inclusión de tres tiempos de análisis, para observar la evolución a lo largo del tiempo de las variables del estudio. Otro de los puntos fuertes incluye el uso de un diseño cruzado a través de path análisis que evalúa la contribución relativa de cada variable a la predicción de las otras variables en el modelo. De esta forma, examinamos tres constructos simultáneamente (estilos inferenciales de desesperanza, victimización entre iguales y sintomatología depresiva) durante la adolescencia, época en la cual estas tres variables desempeñan un papel fundamental en el desarrollo físico y psicológico actual y futuro de los adolescentes (Cova et al., 2012).

A pesar de los puntos fuertes, hubo varias limitaciones que brindan oportunidades para futuras investigaciones. Una de las principales limitaciones es que este estudio ha empleado exclusivamente medidas de autoinforme. A pesar de que los autoinformes constituyen un enfoque confiable para evaluar y conocer los estados emocionales y las cogniciones y son predictores válidos de estados de ánimo, emociones y psicopatologías (Haeffel y Howard, 2010), su uso exclusivo puede contribuir a aumentar las asociaciones estadísticas entre variables, es por ello por lo que sería recomendable de cara a futuras investigaciones acompañar a estos autoinformes de informes elaborados por terceras personas como serían los profesores. Por otro lado, la medida empleada para la victimización entre iguales hace referencia casi únicamente a la violencia psicológica, ya que sólo incluye un ítem de violencia física. Así pues, a pesar de que los estudios explican que la violencia psicológica es la más habitual, sería interesante observar si los resultados del estudio hubieran sido diferentes si se hubieran diferenciado los distintos tipos de victimización entre iguales.

Los resultados obtenidos tienen importantes implicaciones para la intervención en la depresión en los adolescentes. Por un lado, los estilos inferenciales de desesperanza muestran jugar un papel fundamental en el desarrollo de los adolescentes, pudiendo influir de manera negativa en la aparición de determinadas problemáticas como la depresión. Del mismo modo, la sintomatología depresiva genera un empeoramiento de los estilos inferenciales de desesperanza, entrando en un ciclo que se expande en el tiempo. 
Estudios como el actual muestran la necesidad de intervenir con programas de prevención o intervención sobre el desarrollo de estilos inferenciales positivos, permitiendo a los adolescentes mejorar la visión de los diferentes eventos sociales y personales consiguiendo a su vez una disminución de la sintomatología depresiva.

Por otro lado, los resultados indican la necesidad de llevar a cabo programas de prevención e intervención sobre el acoso escolar. La investigación sobre este tema se ha desarrollado notoriamente durante la última década como resultado de las graves consecuencias que ha mostrado tener la victimización entre iguales. Este estudio ha pretendido realizar un análisis de las relaciones existentes entre esta sintomatología depresiva y la victimización entre iguales. Así, corroborando al modelo diátesis-estrés, el estudio actual muestra como la victimización entre iguales predice un aumento de la sintomatología depresiva. Estos hallazgos tienen una gran importancia práctica a la hora de guiar la intervención psicológica con las víctimas y la prevención en toda la población. Así, programas como el KiVa, el programa CIP o el programa Cyberprogram 2.0 han mostrado una gran eficacia en la disminución del bullying en las aulas educativas de múltiples regiones (Cerezo y Sánchez, 2013; Garaigordobil et al., 2017; Mäkelä y Catalán, 2018).

Queda por mencionar que la depresión es uno de los trastornos más comunes en la sociedad actual. La mayoría de las personas que desarrollan depresión han experimentado episodios durante la adolescencia, y muchos adolescentes a pesar de no tener un cuadro de depresión tienen sintomatología común a ella. Es por ello por lo que esta etapa de desarrollo es uno de los momentos más adecuados para llevar a cabo intervenciones con el objetivo de prevenirla, y por tanto reducir o eliminar los efectos tan adversos que esta genera. Así, por último, conviene remarcar que el estudio actual enfatiza la importancia que la depresión, los estilos inferenciales de desesperanza y la victimización entre iguales tienen en la vida y en el desarrollo personal de los adolescentes, y que por tanto, es necesario actuar sobre todas ellas para evitar el ciclo dinámico transaccional y de retroalimentación positiva que puede perpetuarse en un ciclo vicioso entre ellas.

\section{Conflictos de intereses}

Los autores declaran no tener conflictos de intereses.

\section{Referencias}

Abramson, L., Metalsky, G. I. \& Alloy, L. B. (1989). Hopelessness depression: A theory-based subtype of depression. Psychological Review, 96,358-372.https://doi.org/10.1037/0033-295X.96.2.358
Alba, J. y Calvete, E. (2019). Relaciones entre depresión, estrés y esquemas disfuncionales tempranos en adolescentes. Psicología Conductual, 27(2), 183-198.

Alba, J., Calvete, E., Wante, L., Van Beveren, M. \& Braet, C. (2018). Early maladaptive schemas as moderators of the association between bullying victimization and depressive symptoms in adolescents. Cognitive Therapy and Research, 42(1), 24-35. https://doi.org/10.1007/s10608-017-9874-5

Bond, L., Wolfe, S., Tollit, M., Butler, H. \& Patton, G. (2007). A comparison of the Gatehouse bullying scale and the peer relations questionnaire for students in secondary school. The Journal of School Health, 77(2), 75-79. https://doi.org/10.1515/ ijamh-2012-0106

Bowes, L., Joinson, C., Wolke, D. \& Lewis, G. (2016). Peer victimization during adolescence and its impact on depression in early adulthood: Prospective cohort study in the United Kingdom. Journal of Sports Medicine, 50, 176-183. https:// doi.org/10.1136/bmj.h2469

Calvete, E. (2011). Temporal relationships between inferential style and depressive symptoms in adolescents. International Journal of Cognitive Therapy, 4(4), 438-457. https://doi.org/10.1521/ ijct.2011.4.4.438

Calvete, E. (2014). Emotional abuse as a predictor of early maladaptive schemas in adolescents: Contributions to the development of depressive and social anxiety symptoms. Child Abuse \& Neglect, 38(4), 735-746. https://doi.org/10.1016/j. chiabu.2013.10.014

Calvete, E. y Cardeñoso, O. (1999). Creencias y síntomas depresivos: resultados preliminares en el desarrollo de una escala de creencias irracionales abreviada. Anales de Psicología, 15(2), 179-190.

Calvete, E., Fernández-González, L., González-Cabrera, J. M. \& Gámez-Guadix, M. (2018). Continued bullying victimization in adolescents: maladaptive schemas as a mediational mechanism. Journal of Youth and Adolescence, 47(3), 650-660. https://doi. org/10.1007/s10964-017-0677-5

Calvete, E., Orue, I. \& Gámez-Guadix, M. (2015). Cyberbullying victimization and depression in adolescents: the mediating role of body image and cognitive schemas in a one-year prospective study. European Journal on Criminal Policy and Research, 22(2), 271-284. https://doi.org/10.1007/s10610015-9292-8

Calvete, E., Orue, I. \& Hankin, B. L. (2013). Transactional relationships among cognitive vulnerabilities, stressors, and depressive symptoms in adolescence. Journal of Abnormal Child Psychology, 41(3), 399-410. https://doi.org/10.1007/ s10802-012-9691-y

Calvete, E., Orue, I. \& Hankin, B. L. (2015). Cross-lagged associations among ruminative response style, stressors, and depressive symptoms in adolescents. Journal of Social and Clinical Psychology, 34(3), 203-220. https://doi.org/10.1521/ jscp.2015.34.3.203

Calvete, E., Villardón, L. \& Estévez, A. (2008). Attributional style and depressive symptoms in adolescents: an examination of the role of various indicators of cognitive vulnerability. Behaviour Research and Therapy, 46, 944-953. https://doi.org/10.1016/j. brat.2008.04.010

Calvete, E., Villardón, L., Estévez, A. y Espina, M. (2007). La desesperanza como vulnerabilidad cognitiva al estrés. Adaptación 
del cuestionario de estilo cognitivo para adolescentes. Ansiedady estrés. 12(2-3), 215-227.

Cerezo, R. F. y Sánchez, L. C. (2013). Eficacia del programa CIP para la mejora de la convivencia escolar y la prevención del bullying en alumnos de Educación Primaria. Apuntes de Psicología, 31(2), 173-181.

Chocarro, E. \& Garaigordobil, M. (2019). Bullying y cyberbullying: diferencias de sexo en víctimas, agresores y observadores. Pensamiento Psicológico, 17(2), 57-71. https://doi.org/10.11144/ doi:10.11144/Javerianacali.PPSI17-2.bcds

Cole, D. A., Ciesla, J. A., Dallaire, D. H., Jacquez, F. M., Pineda, A. Q., LaGrange, B., Truss, A. E., Folmer, A. S., Tilghman-Osborne, C. \& Felton, J. W. (2008). Emergence of attributional style and its relation to depressive symptoms. Journal of Abnormal Psychology, 117(1), 16-31. https://doi.org/10.1037/0021-843X.117.1.16

Cole, D. A., Zelkowitz, R. L., Nick, E., Martin, N. C., Roeder, K. M., Sinclair-McBride, K. \& Spinelli, T. (2016). Longitudinal and incremental relation of cybervictimization to negative self-cognitions and depressive symptoms in young adolescents. Journal of Abnormal Child Psychology, 44(7), 1321-1332. https://doi.org/10.1007/s10802-015-0123-7

Cova, F., Rincón, P., Inostroza, C. y Melipillán, R. (2012). Factores asociados prospectivamente a sintomatología depresiva en adolescentes tempranos de sexo femenino. Universitas Psychologica, 11(3), 853-862.

Davoodi, E., Wen, A., Dobson, K., Noorbala, A., Mohammadi, A. \& Farahmand, Z. (2018) Early maladaptive schemas in depression and somatization disorder. Journal of Affective Disorders, 235 , 82-89. https://doi.org/10.1016/j.jad.2018.04.017

Delgado, B. \& Escortell, R. (2018). Sex and grade differences in cyberbullying of spanish students of 5th and 6th grade of primary education. Anales de Psicología, 34(3), 472-481. https://doi.org/10.6018/analesps.34.3.283871

Garaigordobil, M., Martínez-Valderrey, V. y Machimbarrena, J. (2017). Intervención en el bullying y ciberbullying: Evaluación del caso Martín. Revista de Psicología Clínica con Niños y adolescentes, 4(1), 25-32.

Gibb, B. E. \& Abela, J. R. Z. (2008). Emotional abuse, verbal victimization, and the development of children's negative inferential styles and depressive symptoms. Cognitive Therapy and Research, 32, 161-176. https://doi.org/10.1007/s10608006-9106-x

Gómez-Odriozola, J. \& Calvete, E. (2020). Longitudinal bidirectional associations between dispositional mindfulness, maladaptive schemas, and depressive symptoms in adolescents. Mindfulness, 11, 1943-1955. https://doi.org/10.1007/s12671-020-01402-w

Haeffel, G. J. \& Howard, G.S. (2010). Self-report: Psychology's four-letter word. The American Journal of Psychology, 123(2), 181-188. https://doi.org/10.5406/amerjpsyc.123.2.0181

Hammen, C. (1991). Generation of stress in the course of unipolar depression. Journal of Abnormal Psychology, 100(4), 555561. https://doi.org/10.1037/0021-843X.100.4.555

Hammen, C. (2005). Stress and depression. Annual Review of Clinical Psychology, 1(1), 293-319. https://doi.org/10.1146/ annurev.clinpsy.1.102803.143938

Hankin, B. L. (2008). Stability of cognitive vulnerabilities to depression: A short-term prospective multiwave study. Journal of Abnormal Psychology, 117(2), 324-333. https:// doi.org/10.1037/0021-843X.117.2.324
Hankin, B. L. (2010). Personality and depressive symptoms: stress generation and cognitive vulnerabilities to depression in a prospective daily diary study. Journal of Social and Clinical Psychology, 29(4), 369-401. https://doi.org/10.1521/ jscp.2010.29.4.369

Hankin, B. L. \& Abramson, L.Y. (2001). Development of gender differences in depression: an elaborated cognitive vulnerabilitytransactional stress theory. Psychological Bulletin, 127(6), 773-796. https://doi.org/10.1037/0033-2909.127.6.773

Hankin, B. L. \& Abramson, L. Y. (2002). Measuring cognitive vulnerability to depression in adolescence: reliability, validity, and gender differences. Journal of Clinical Child and Adolescent Psychology, 31(4), 491-504. https://doi. org/10.1207/153744202320802160

Hankin, B. L., Abramson, L. Y., Moffitt, T. E., Silva, P. A., McGee, R. \& Angell, K. E. (1998). Development of depression from preadolescence to young adulthood: emerging gender differences in a 10-year longitudinal study. Journal of Abnormal Psychology, 107(1), 128-140. https://doi.org/10.1037/0021843X.107.1.128

Hu, L. \& Bentler, P. M. (1999). Cutoff criteria for fit indexes in covariance structure analysis: conventional criteria versus new alternatives. Structural Equation Modeling, 6(1), 1-55. https://doi.org/10.1080/10705519909540118

Hymel, S. \& Swearer, S. M. (2015). Four decades of research on school bullying: An introduction. American Psychologist, 70(4), 293-299. https://doi.org/10.1037/a0038928

Janovsky, T., Rock, A. J., Thorsteinsson, E. B., Clark, G. I. \& Murray, C. V. (2020). The relationship between early maladaptive schemas and interpersonal problems: A metaanalytic review. Clinical Psychology \& Psychotherapy, 27(3), 408-447. https://doi.org/10.1002/cpp.2439

Krause, E. D., Vélez, C. E., Woo, R., Hoffmann, B., Freres, D. R., Abenavoli, R. M. \& Gillham, J. E. (2018). Rumination, depression, and gender in early adolescence: a longitudinal study of a bidirectional model. The Journal of Early Adolescence, 38(7), 923-946. https://doi.org/10.1177/0272431617704956

Lewinsohn, P. M., Steinmetz, J. L., Larson, D. W. \& Franklin, J. (1981). Depression-related cognitions: antecedent or consequence? Journal of Abnormal Psychology, 90(3), 213-219. https://doi. org/10.1037/0021-843X.90.3.213

Little, J. (2013). Multilevel confirmatory ordinal factor analysis of the Life Skills Profile-16. Psychological Assessment, 25(3), 810-825. https://doi.org/10.1037/a0032574

Mac Giollabhui, N., Hamilton, J. L., Nielsen, J., Connolly, S. L., Stange, J. P., Varga, S., Burdette, E., Olino, T. M., Abramson, L. Y. \& Alloy, L. B. (2018). Negative cognitive style interacts with negative life events to predict first onset of a major depressive episode in adolescence via hopelessness. Journal of Abnormal Psychology, 127(1), 1-11. https://doi.org/10.1037/ abn0000301

Mäkelä, T. y Catalán, B. L. (2018). Programa de convivencia y anti-acoso escolar KiVa: Impacto y reflexión. Anales de la Fundación Canis Majoris, 2, 234-258.

Marshall, S. K., Tilton-Weaver, L. C. \& Stattin, H. (2013). Nonsuicidal self-injury and depressive symptoms during middle adolescence: a longitudinal analysis. Journal of Youth Adolescence, 42, 1234-1242. https://doi.org/10.1007/s10964013-9919-3 
Masten, A. S., Roisman, G. I., Long, J. D., Burt, K. B., Obradović, J., Riley, J. R., Boelcke-Stennes, K. \& Tellegen, A. (2005). Developmental cascades: Linking academic achievement and externalizing and internalizing symptoms over 20 years. Developmental Psychology, 41(5), 733-746. https://doi. org/10.1037/0012-1649.41.5.733

Mateos-Pérez, E. y Calvete, E. (2019). Interacción entre diferentes factores cognitivos en la predicción de los síntomas de depresión. Psicología Conductual, 27(1), 21-39.

Moore, S. E., Norman, R. E., Suetani, S., Thomas, H. J., Sly, P. D. \& Scott, J. G. (2017). Consequences of bullying victimization in childhood and adolescence: A systematic review and metaanalysis. World Journal of Psychiatry, 7(1), 60-76. https:// dx.doi.org/10.5498\%2Fwjp.v7.i1.60

Pouwels, J. L., Souren, P. M., Lansu, T. A. M. \& Cillessen, A. H. N. (2016). Stability of peer victimization: A meta-analysis of longitudinal research. Developmental Review, 40, 1-24. https://doi.org/10.1016/j.dr.2016.01.001

Radloff, L. S. (1977). The CES-D Scale: a self-report depression scale for research in the general population. Applied Psychological Measurement, 1(3), 385-401. https://doi. org/10.1177/014662167700100306

Rigby, K. \& Slee, P.T. (1993). Dimensions of interpersonal relation among Australian children and implications for psychological wellbeing. Journal of Social Psychology, 133(1), 33-42. https://doi.org/10.1080/00224545.1993.9712116

Rose, D. T. \& Abramson, L. Y. (1992). Developmental predictors of depressive cognitive style: Research and theory. En D. Cicchetti y S. Toth (Eds.), Rochester symposium of developmental psychopathology, Vol. IV (pp. 323-349). University of Rochester Press.

Saint-Georges, Z. \& Vaillancourt, T. (2020). The temporal sequence of depressive symptoms, peer victimization, and self-esteem across adolescence: Evidence for an integrated self-perception driven model. Development and Psychopathology, 32(3), 975-984. https://doi.org/10.1017/ S0954579419000865

Salk, R. H., Hyde, J. S. \& Abramson, L. Y. (2017). Gender differences in depression in representative national samples: meta-analyses of diagnoses and symptoms. Psychological Bulletin, 143(8), 783-822. https://doi.org/10.1037/bul0000102

Silva, J., Oliveira, W., Komatsu, A., Zequiñao, M., Pereira, B., Caravita, S., Skrzypiec, G. \& Silva, M. (2020). Associations between bullying and depression among students in school transition. Trends in Psychology, 28, 72-84. https://doi. org/10.1007/s43076-020-00017-3

Sociedad Española de Epidemiología y Medicina Familiar y Comunitaria. (2000). Una propuesta de medida de la clase social. Atención Primaria, 25(5), 350-363. https://doi. org/10.1016/S0212-6567(00)78518-0

Sweeting, H., Young, R., West, P. \& Der, G. (2006). Peer victimization and depression in early-mid adolescence: A longitudinal study. British Journal of Educational Psychology, 76(3), 577-594. https://doi.org/10.1348/000709905X49890

Zhang, D., Huebner, E. S. \& Tian, L. (2020). Longitudinal associations among neuroticism, depression, and cyberbullying in early adolescents. Computers in Human Behavior, 112. https://doi.org/10.1016/j.chb.2020.106475 